\title{
Endoscopic closure of a fistula between an ileal conduit and an ileal handle localized between two uretero-ileal anastomoses
}

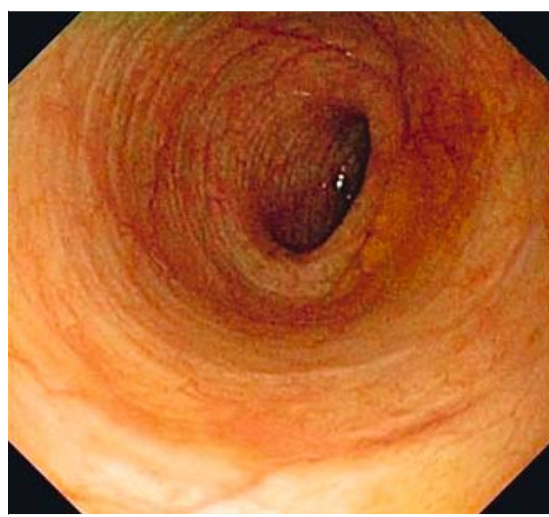

- Fig. 1 Endoscopic view of the ileal conduit.

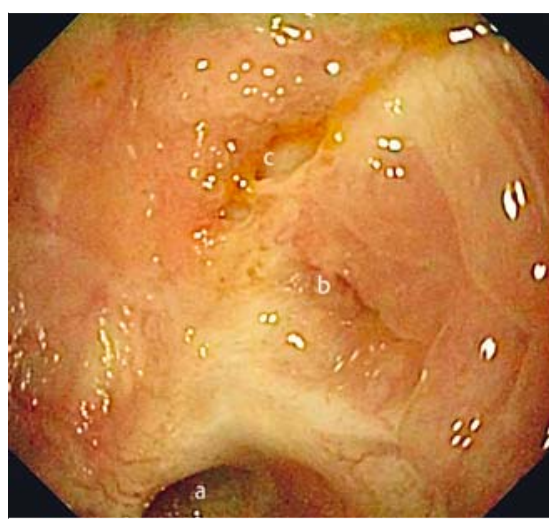

- Fig. 2 Endoscopic view of the fistula (c) between the right (a) and left (b) ureteroileal anastomoses.

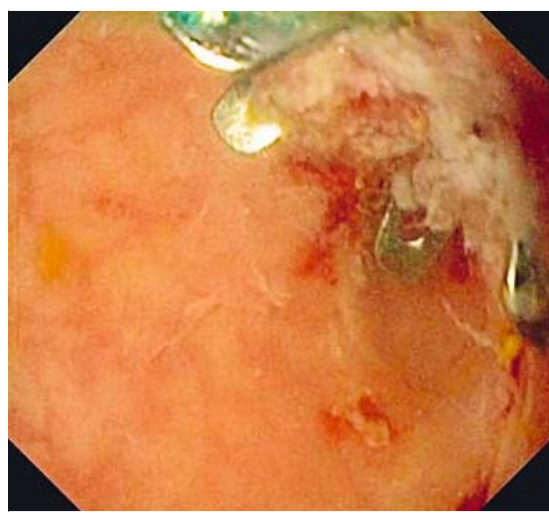

Fig. 3 An over-the-scope clip was deployed to close the leak.
Cystectomy is the gold standard treatment for patients with bladder cancer. Urinary diversion with ileal conduit and uretero-ileal anastomoses, as described by Bricker, is the most widely used surgical therapy because of the lower risk of postoperative complications in elderly patients and in those with co-morbidities. The Bricker technique involves the use of a segment of the ileum as a conduit to the skin, with a successive uretero-ileal-cutaneous anastomosis for each ureter [1]. The endoscopic approach to construction of the ileal conduit for urological obstruction is rarely reported [2]. We present the case of a patient who underwent cystectomy with a Bricker uretero-ileal-cutaneous anastomosis, who developed a fistula between the ileal conduit and an ileal handle.

In May 2015, the patient underwent cystectomy with a Bricker uretero-ilealcutaneous anastomosis because of bladder transitional cell carcinoma. In October 2016, stool appeared in the drainage. The patient underwent radiological examination with contrast medium at another hospital, which revealed a fistula between the ileal conduit and an ileal handle. The patient was referred to our unit and an ileal conduit endoscopy ( $\triangleright$ Fig. 1) was performed using a gastroscope, which showed stool leakage from an orifice between the two ureteral anastomoses ( $\triangleright$ Fig. 2 ). An 11/6 traumaticteeth over-the-scope clip (OTSC), $9 \mathrm{~mm}$ in diameter, was placed to close the leak ( $\vee$ Video 1), using an OTSC anchor to grasp the fistula ( $\triangleright$ Fig.3). Stool no longer appeared in the drainage 24 hours after OTSC placement. No adverse events occurred, and the patient was discharged 3 days after the procedure.

There are no reports in the literature of the endoscopic closure of a fistula between the ileal conduit and an ileal handle. The current case demonstrates successful closure using an OTSC, which avoided damage to the uretero-ileal anastomoses. The OTSC is an excellent endoscopic therapeutic and conservative option in this particular and rare adverse event.

Endoscopy_UCTN_Code_TTT_1AQ_2AG

\section{Competing interests}

None

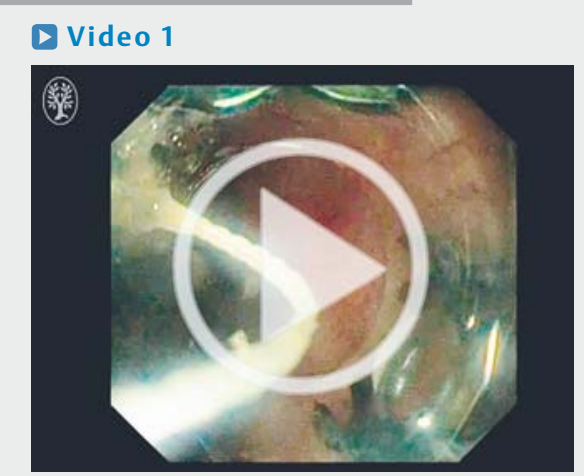

- Video 1: Placement of an over-the-scope clip to seal the fistula between the ileal conduit and an ileal handle, which was located between the two ureteral anastomoses. 
The Authors

Benedetto Mangiavillano ${ }^{1}$, Mario Bianchetti ${ }^{1}$, Loretta Amato', Sara Melegari², Mauro Seveso $^{2}$, Gianluigi Taverna², Alessandro Repici ${ }^{3}$

1 Gastrointestinal Endoscopy Unit, Humanitas Mater Domini, Castellanza, Italy

2 Urology Unit, Humanitas Mater Domini, Castellanza, Italy

3 Digestive Endoscopy Unit, Istituto Clinico Humanitas Research Hospital, Milan, Italy
Corresponding author

Benedetto Mangiavillano, MD

Gastrointestinal Endoscopy Unit, Humanitas

- Mater Domini, Via Gerenzano n.2, 21053 -

Castellanza (VA), Italy

Fax: +39-0331-476372

b_mangiavillano@hotmail.com

\section{References}

[1] Bricker EM. Substitution for the urinary bladder by use of isolated ileal segments. Surg Clin North Am 1956; 36: 1117-1128

[2] Mangiavillano B, Montanari E, Santoro T et al. Double endoscopic retrograde ureteral drainage performed with a standard gastroscope in a cystectomized patient with a Wallace ureteroilealcutaneous anastomosis. Dig Liver Dis 2012; 44: 624-625

\section{Bibliography}

DOI http://dx.doi.org/10.1055/s-0043-103403 Endoscopy 2017; 49: E125-E126

(c) Georg Thieme Verlag KG

Stuttgart · New York

ISSN 0013-726X 